\title{
ARTICLE
}

\section{Forward and adjoint Monte Carlo simulations for sensitivity studies in difficult geometries}

\author{
Dorothea Sommer ${ }^{*}$, Uwe Reichelt, Marian Sommer and Jürgen Henniger \\ Technische Universität Dresden, Institute for Nuclear and Particle Physics, Helmholtzstr. 10, Dresden, 01062, Germany
}

\begin{abstract}
Integral dose measurement is proposed for release measurement in inaccessible geometries like ducts and pipes. The initial steps for the feasibility test of this method are presented in this paper on the example of a pipe in a large concrete element. Extensive Monte Carlo simulations are carried out, which are a sensitivity study towards the different influences on the possible dose rates from different activity distributions. With distances between the measurement points of $10 \mathrm{~cm}$, the detection of release value contaminations is possible for all constellations.
\end{abstract}

\section{Keywords: AMOS; adjoint photon transport; release measurement; contamination}

\section{Introduction}

At the dismantling of nuclear facilities, the common strategy is to come out with as little as possible debris for special disposal. Also, the main demolition work should be carried out at elements released from nuclear safety regulations. Thus, there is the need to accomplish well-founded release measurements also at points, which are difficult to access but could still be contaminated, without having to modify the construction element. Typical for such inaccessible positions are pipes, cavities, and gaps which cannot be examined by common in-situ measurements.

To provide a solution for this problem, integral dose measurement with small dosimeters, which could be TL or OSL detectors, is being considered. This method would have two advantages. Small dosimeters can be inserted in even narrow ducts and pipes within large construction elements. Furthermore, as many detectors as required can be brought up and, after exposure, recollected and evaluated in short time.

The main challenge of this method is to distinguish between the dose due to the natural radioactivity of the surrounding material and the dose originating from contaminations with activities in the range of the release value of the nuclide.

Thus, it is the task of this paper to computationally proof, that it is possible to conservatively detect release value contaminations with an integrating measurement method. Preparatory calculations are presented to show the feasibility of this method as well as to give an example how to deliver calibration factors for special geometries.

\section{Material and methods}

\subsection{Geometry}

Geometry framework is a straight pipe within a large concrete block. The pipe is of outer $\varnothing 26.9 \mathrm{~mm}$ and wall thickness of 2.65 mm PVC, see Figure 1.

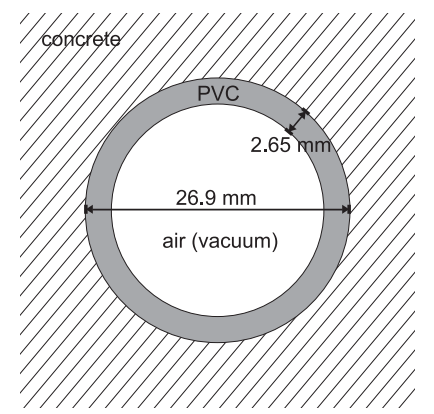

Figure 1. Lateral cut through the PVC pipe embedded in concrete.

Natural activity of concrete strongly depends on the origin of the ingredient materials. Values for the levels relevant for Germany of the Radium (Uranium) and Thorium series and of ${ }^{40} \mathrm{~K}$ are listed in Table $\mathbf{1}$.

Table 1. Specific activity of natural radionuclides in concrete in $\mathrm{Bq} / \mathrm{kg}$ [2].

\begin{tabular}{lccr}
\hline & Radium series & Thorium series & ${ }^{40} \mathrm{~K}$ \\
\hline medium & $\mathbf{3 0}$ & $\mathbf{2 3}$ & $\mathbf{4 5 0}$ \\
minimum & 7 & 4 & 50 \\
maximum & 92 & 71 & 1300 \\
\hline
\end{tabular}

*Corresponding author. Email: d.sommer@asp.tu-dresden.de 


\subsection{Monte Carlo calculations}

The Monte Carlo code AMOS [2,3] is used to estimate fluence spectra in the pipe. These are convolved with the fluence to Air Kerma conversion coefficients from [4] to estimate the dose rate. For a special dosimetry system, the energy dependent dose response should be included at this point.

The simulations concerning the dose due to the natural background imply a large source volume while the fluence has to be estimated for a small detecting volume inside the narrow pipe. This is an inherent adjoint problem, so they are carried out with the adjoint photon kernel of the AMOS code. The contamination situations are calculated in the forward mode as both, source and detector volume, are small. For this constellation, the forward simulation is usually faster.

The source term is defined according to [5], while emissions with probabilities below 1E-8 are disregarded. Alphas will not contribute to the measurement effect and are therefore ignored. The same holds for electrons as long as the detector cover is thick enough to stop them. Nevertheless, a secondary photon source term resulting mainly from X-Ray deexcitations but also from secondary bremsstrahlung from beta and electron emissions is calculated for homogeneous concrete. The outcome spectrum in photons per energy per decay is utilized as source term in addition to the discrete gamma emissions for the pure photon simulations. As an example, Figure 2 shows the resulting photon spectrum emitted from ${ }^{40} \mathrm{~K}$.

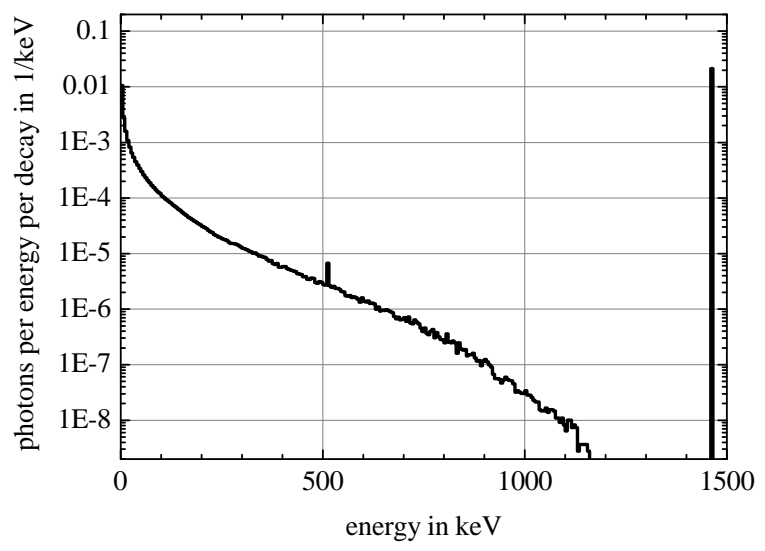

Figure 2. Total spectral photon emissions per decay $40 \mathrm{~K}$ in concrete (5 keV binning).

\section{Results}

\subsection{Infinite concrete}

Firstly, a very simple geometry is considered. The Air Kerma at a point in infinite concrete is estimated. The source, the natural activity of the concrete, is uniformly distributed within spheres of different radii from this point.

Due to the geometrical symmetry, this simple problem can be calculated adjoint and forward in the same manner. The forward calculation involves nuclide data down to emission probabilities of $1 \mathrm{E}-8$ and complete electron simulation.

In Figure 3, the amounts of Air Kerma rate induced by $1 \mathrm{~Bq} / \mathrm{cm}^{3}$ (mother) nuclide from the natural radioactivity are compared. Forward and adjoint results yield a difference only in the range of the calculation uncertainty of $3 \%$. Thus, it shows that the secondary photon source term can be used as a good approximation for the pure photon simulation of radiation fields due to nuclide decay.

Figure 4 nets the contributions of the natural radio nuclides via the medium specific activity in concrete of Table $1.99 \%$ of the dose rate originates in depths less than $50 \mathrm{~cm}$. So, if the investigated concrete block covers the pipe with significantly less than $50 \mathrm{~cm}$, a special calibration is required.

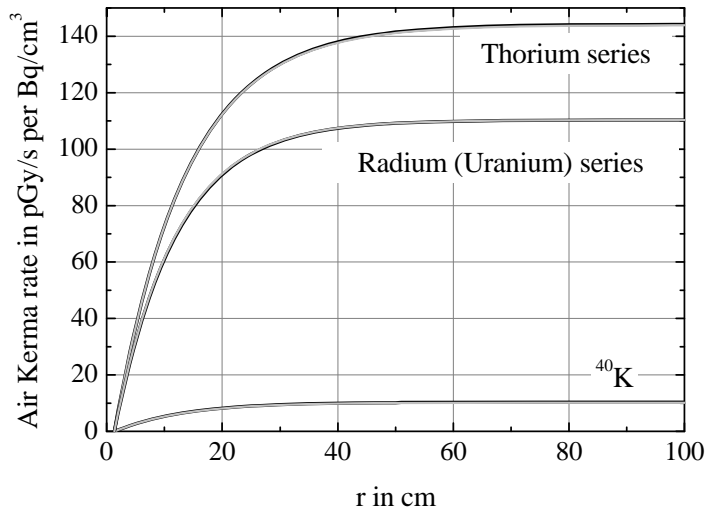

Figure 3. Air Kerma rate due to activity concentration of (mother) nuclide uniformly distributed in spheres of radius $r$. Black - forward simulation, grey - adjoint simulation.

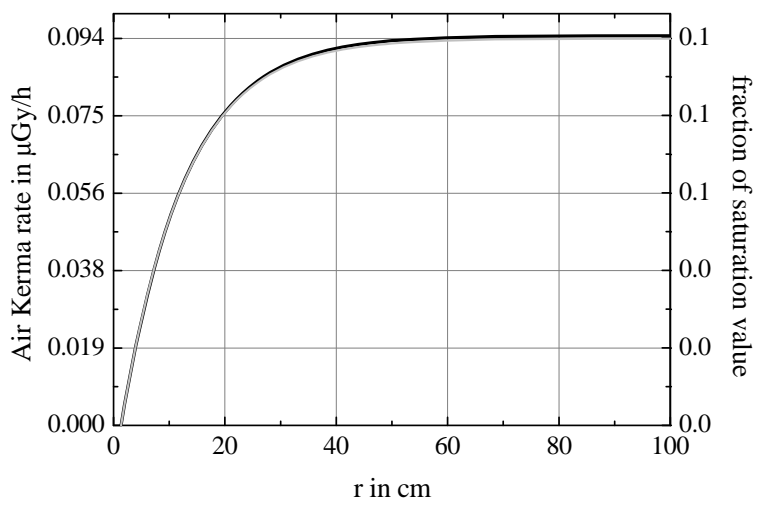

Figure 4. Air Kerma rate due to the medium specific activity of natural radionuclides in concrete uniformly distributed in spheres of radius $r$. Black - forward simulation, grey - adjoint simulation.

\subsection{Pipe geometry - natural background}

For all further calculations, the PVC pipe geometry is used. Table 2 lists the calculated background dose rates due to the natural activity of the surrounding concrete per activity concentration. 
Table 2. Air Kerma in pGy/s in the PVC pipe due to $1 \mathrm{~Bq} / \mathrm{cm}^{3}$ of natural radionuclides in the surrounding concrete.

\begin{tabular}{ccc}
\hline Radium series & Thorium series & ${ }^{40} \mathrm{~K}$ \\
\hline 109 & 148 & 11.0 \\
\hline
\end{tabular}

Thus, if the medium natural specific activity (see Table 1) is assumed, the surrounding concrete would lead to a dose rate of $0.096 \mu \mathrm{Gy} / \mathrm{h}$.

One problem in practice that must not be disregarded is the Radon which migrates through the concrete. The Radon reaching the pipe will stay inside and either concentrate there or it will flow downwards to the lowest point. Both cases would lead to an increase of background dose rate, the latter rather significantly but locally. To avoid this, the pipe should be ventilated before and during the measurement time.

It is not avoidable to have an outlet of the pipe. So, if the detectors are placed closer to this outlet, the background dose rate will reduce. Figure 5 shows this reduction in dependence of the distance $\mathrm{z}$ to the outlet inside the concrete. The maximum of $0.096 \mu \mathrm{Gy} / \mathrm{h}$ is again reached at approx. $50 \mathrm{~cm}$, and the dose rate decreases down to less than $50 \%$ as the measurement point approaches the outlet at $\mathrm{z}=0 \mathrm{~cm}$.

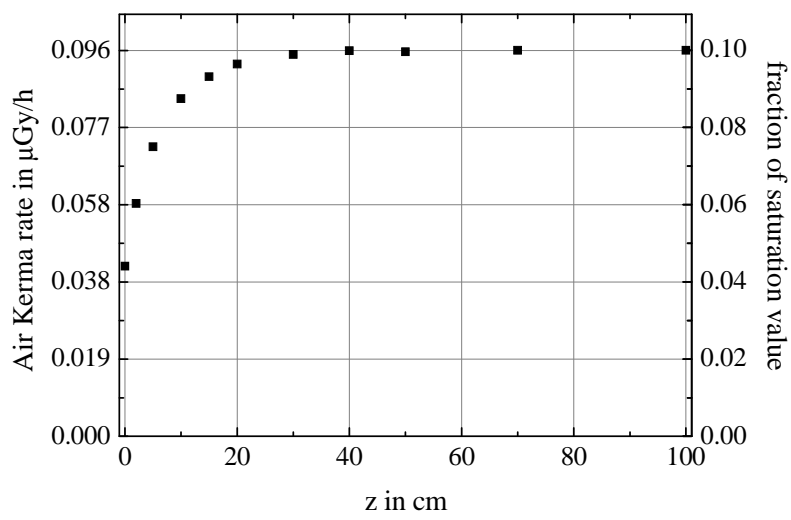

Figure 5. Background dose rate when approaching the outlet of the PVC pipe at $\mathrm{z}=0 \mathrm{~cm}$.

\subsection{Pipe geometry - contaminations}

Some assumptions have to be made before considering different contaminations. Firstly, it is understood that the activity found its way to the examination point only through the pipe itself. The second is that any wipeable remainder was extracted from the pipe. What stays are not detachable contaminations on the inner surface of the pipe which could, in theory, have any shape.

The question is now about the dose rate distribution inside the pipe. In principle, if the measurement is carried out in the cylindrical symmetry, any dose rate distribution due to an activity distribution is just the integral over the point source solution. So, it is sufficient to calculate the dose rates inside the pipe coming from a point contamination on its inner surface.

The later positions of the detectors will be attempted to be on the cylinder axis. But as they somehow have to be brought inside, there will always be some unavoidable displacement. Thus, it is reasonable for the simulation to average over the inner cross-sectional area. So, the detection volumes for the dose rate simulation for the following problems will be thin slices of the inner pipe.

Summarizing, a point contamination on the inner surface of the PVC pipe is considered for the following calculations. As examples, the contamination is assumed to be ${ }^{137} \mathrm{Cs}$ or ${ }^{60} \mathrm{Co}$. The Air Kerma is calculated for thin lateral slices inside the pipe at different z-distances from the contamination. Figure 6 gives an impression of this setup as longitudinal cut through the PVC pipe not including the detector volumes.
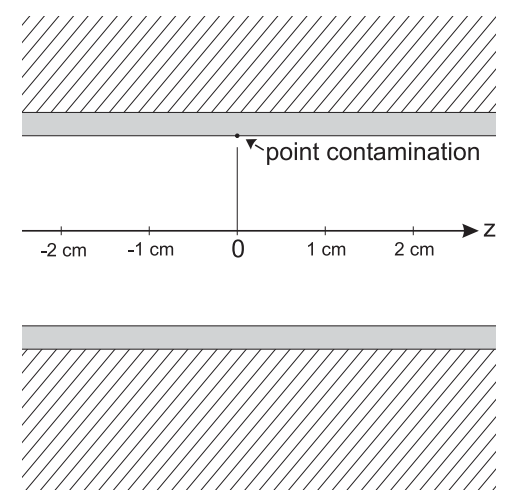

Figure 6. Longitudinal cut through the PVC pipe with contamination at $\mathrm{z}=0 \mathrm{~cm}$.

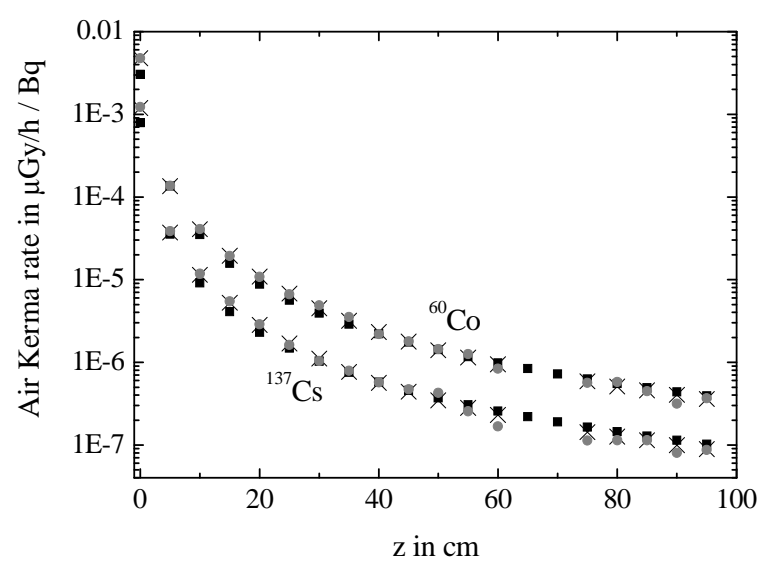

Figure 7. Air Kerma rate in different distances $\mathrm{z}$ from a point contamination at $\mathrm{z}=0 \mathrm{~cm}$. Black squares - dose rate factors of the nuclides, grey dots - complete forward simulation, black crosses - pure photon forward simulation.

In Figure 7, the outcome of the simulation is presented. To give comparison values, the dose rate factors of the nuclides, listed in Table 3, in combination with the inverse square law are applied for a simple calculation. For these photon energies, Air Kerma and equivalent dose do not greatly differ, which makes the comparison possible. The calculation is carried out for 
Table 3. Dose rate factors for nuclides in $\mathrm{mSv} / \mathrm{h} \mathrm{m}^{2} / \mathrm{GBq}$ [6].

\begin{tabular}{cc}
\hline${ }^{60} \mathrm{Co}$ & ${ }^{137} \mathrm{Cs}$ \\
\hline 0.354 & 0.0927 \\
\hline
\end{tabular}

points in the centre of the pipe.

In Figure 7, the difference between simulation result and calculation by means of the dose rate factors at $\mathrm{z}=0 \mathrm{~cm}$ originates from the different detector volumes. At this proximity to the source, the inverse square law has a large influence. Up to $40 \mathrm{~cm}$ distance, scattering in the material, PVC as well as nearby concrete, slightly heightens the dose rate. Further out than $50 \mathrm{~cm}$, the simulation has a large uncertainty due to the very inefficient geometry.

To estimate the sensitivity of an integrating measurement system, the background dose rate from the natural activity and the dose rate from possible contaminations have to be combined. As stated, integration of the dependency displayed in Figure 7 yields the information about dose rates from any contamination distribution on the inner surface of the PVC pipe.

In German law, the release values for radionuclides are listed in the radiation protection law [7] in App. III, Table 1. For buildings projected for demolition, column 10 is to be applied, which gives surface contamination release values of $3 \mathrm{~Bq} / \mathrm{cm}^{2}$ for ${ }^{60} \mathrm{Co}$ and of $10 \mathrm{~Bq} / \mathrm{cm}^{2}$ for ${ }^{137} \mathrm{Cs}$. It is allowed to average the corresponding measurement over $1 \mathrm{~m}^{2}$, but this makes only little sense regarding the here discussed geometry.

With the pipe of diameter of $2.16 \mathrm{~cm}, 20 \mathrm{~cm}$ length gives an inner surface of about $136 \mathrm{~cm}^{2}$. Assuming this as averaging area, the total release values are $407 \mathrm{~Bq}$ of ${ }^{60} \mathrm{Co}$ or $1357 \mathrm{~Bq}$ of ${ }^{137} \mathrm{Cs}$. Now, it has to be proven, that any distribution of this amount of activity can be detected with the proposed measurement method.

Figures 8 and 9 show dose rate contours for different distributions of the release value contaminations of ${ }^{60} \mathrm{Co}$ and ${ }^{137} \mathrm{Cs}$, respectively.

It is now proposed that the dosimeters are placed in the pipe with $10 \mathrm{~cm}$ distance between each other. Then, the worst case situation to detect a contamination above the release value would be a point contamination which lies directly in between two detectors. As can be seen in Figures 8 and 9, this setup would lead to an increase of measurement effect of about $50 \%$ above the natural background for those two dosimeters. Thus, measurement outputs at two neighboring points higher than this value could indicate a point contamination of higher than the release value.

So, for conservative release measurement of possible ${ }^{137} \mathrm{Cs}$ contamination in this special geometry, $50 \%$ dose enhancement at two neighboring measurement points should be appointed as one criterion for the violation of the release value.

Figure 10 shows the scaled down dose rate contours of the spread out contamination to meet the dose rate of the point contamination in $+/-5 \mathrm{~cm}$. The scaling factor

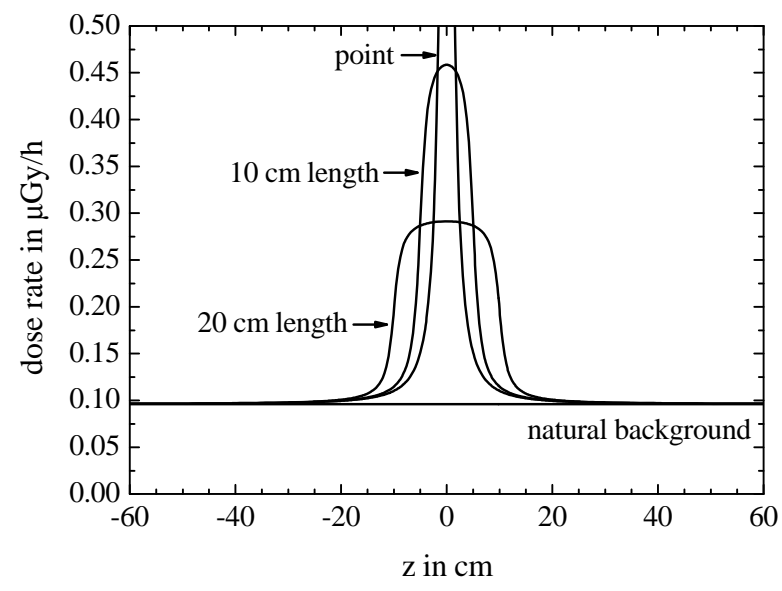

Figure 8. Dose rate in the centre of the PVC pipe due to natural background and surface contaminations of $407 \mathrm{~Bq}{ }^{60} \mathrm{Co}$ of different distributions.

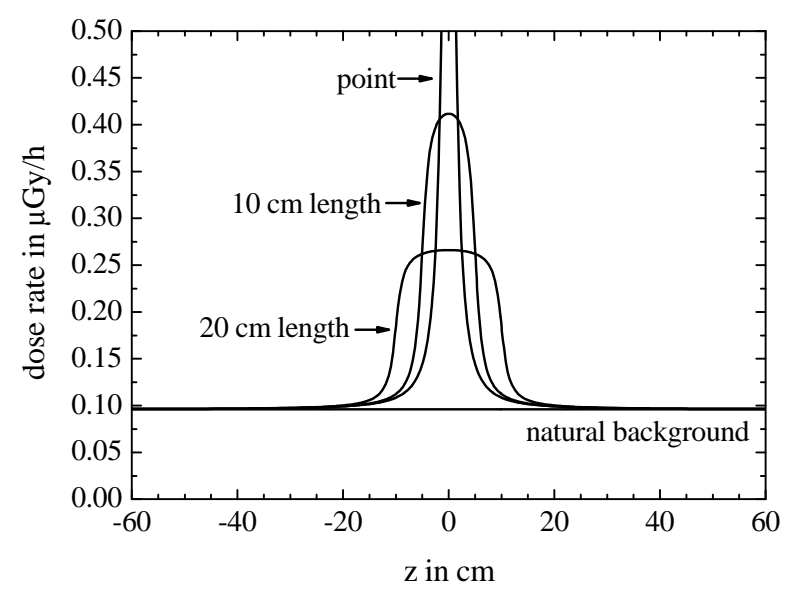

Figure 9. Dose rate in the centre of the PVC pipe due to natural background and surface contaminations of $1357 \mathrm{~Bq}$ ${ }^{137} \mathrm{Cs}$ of different distributions.

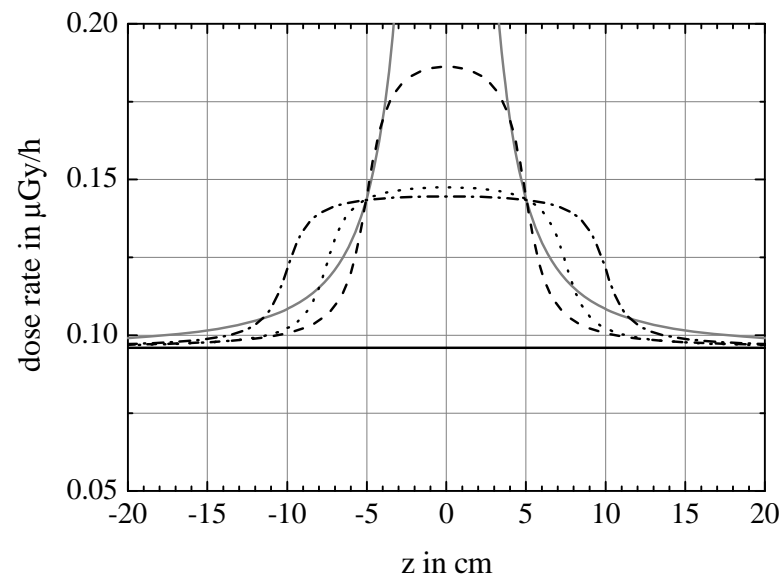

Figure 10. Scaled dose rate profile of ${ }^{137}$ Cs. Grey - point contamination, lines $-10 \mathrm{~cm}$ length, line-dotted $-15 \mathrm{~cm}$ length, dotted - $20 \mathrm{~cm}$ length. 
reaches its maximum at approx. $13 \mathrm{~cm}$ where its value is 4.5. Looking at the position of $+/-15 \mathrm{~cm}$ where the next detectors should be positioned it becomes clear that the overshoot of this criterion is such, that if instead the activity is distributed over a length, it could not be distinguished from the point case.

As ${ }^{137} \mathrm{Cs}$ leads to a lower dose rate increase compared to ${ }^{60} \mathrm{Co}$ but with the same shape, this discussion can be adopted for ${ }^{60} \mathrm{Co}$ with slightly lower values.

\section{Conclusion}

The problem of release measurement in difficult geometries as ducts and channels in massive concrete is approached by the idea of integral dose measurement with many small dosimeters. In this paper, the plausibility of such measurements is tested for the geometry of a PVC pipe in solid concrete. Monte Carlo simulations are applied to give information about dose rates inside the pipe. The background dose rate due to the natural activity of the concrete is compared with the dose rate profile from ${ }^{60} \mathrm{Co}$ and ${ }^{137} \mathrm{Cs}$ contamination on the inner surface. To calculate the first, only the adjoint mode of the Monte Carlo code is reasonable to apply by means of the geometry composition. To still completely simulate the nuclear decay by means of pure photon transport, in addition to the discrete gamma emissions, a secondary photon source term is established which accounts for X-Ray emissions of the atoms and secondary bremsstrahlung from betas. Good results are achieved with this method.

Overall, the conclusion is that with measurement points of $10 \mathrm{~cm}$ distance and an averaging area of $136 \mathrm{~cm}^{2}$, which corresponds to $20 \mathrm{~cm}$ of pipe length, contaminations above the appropriate release value can easily be detected. The most conservative criterion, which takes into account a possible point contamination, leads to a maximum overshoot of a factor of 4.5 for spread out contamination.

\section{Acknowledgements}

The computations were performed on an HPC-Windows-Cluster Titan at the Center for Information Services and High Performance Computing (ZIH) at the TU Dresden.

\section{References}

[1] Bundesamt für Strahlenschutz (BfS), Umweltradioaktivität und Strahlenbelastung: Jahresbericht 2005, (2006).

[2] U. Reichelt and J. Henniger, Application of advanced Monte Carlo methods in numerical dosimetry, Radiat. Protect. Dosim. 119 (2006), pp. 479-482.

[3] D. Gabler, J. Henniger and U. Reichelt, AMOS An effective tool for adjoint Monte Carlo simulations, Nucl. Instrum. Meth. B 251 (2006), pp. 326-332.

[4] ICRP 74 - Conversion Coefficients for use in Radiological Protection against External Radiation, Annal of the ICRP 26/3 (2007).

[5] Evaluated Nuclear Structure Data File (ENSDF), ENSDF Database Manager: Jag Tuli, NNDC, Brookhaven National Laboratory; Data Source: ENSDF is compiled by the International Network of Nuclear Structure and Decay Data Evaluators (http://www-nds.iaea.org/nsdd/).

[6] DIN6844-3: Nuclear medicine departments - Part 3: Radiation protection calculations, Deutsches Institut für Normung, (2006).

[7] Verordnung über den Schutz vor Schäden durch ionisierende Strahlen (Strahlenschutzverordnung StrlSchV), (24.2.2012). 